\title{
Patrones de Usabilidad Temprana en el Modelo Conceptual
}

\section{Early Usability Patterns in the Conceptual Model}

Presentación: 28/08/2020

\section{Doctorando:}

\section{Juan Carlos Moreno}

Centro de Investigación, Desarrollo y Transferencia de Sistemas de Información (CIDS). Facultad Regional Córdoba. Universidad Tecnológica Nacional. Argentina

jmoreno33@gmail.com

\section{Director/es:}

\section{Marcelo Martín Marciszack}

\section{Co-director:}

\section{Mario Alberto Groppo}

\section{Resumen}

Internet ha incrementado el desarrollo y uso de aplicaciones en entorno web. Sin embargo, no todas las aplicaciones web tienen éxito en el mercado. Muchos sistemas son desarrollados con fallas en la usabilidad, que son muy costosas de arreglar una vez que el sistema fue construido. La usabilidad de un sistema de información determina el grado de satisfacción que experimenta un usuario al interactuar con el mismo. Por este motivo, la Usabilidad está fuertemente ligada al ciclo de vida del software y es necesario incorporarla como una característica de calidad a tener en cuenta, durante todo el proceso de desarrollo. Una forma de lograr este objetivo es a través de los patrones de usabilidad. La Ingeniería de Sistemas de Información ha incrementado el empleo de patrones en el modelado conceptual de los sistemas para garantizar no cometer errores y emplear buenas prácticas aprendidas. La siguiente propuesta muestra un conjunto de patrones de usabilidad que pueden incorporarse en el modelado conceptual de los sistemas en forma temprana, para garantizar la presencia de ciertos criterios de Usabilidad desde la etapa de elicitación de los requerimientos.

Palabras clave: Patrón; Modelo de Conceptual; Requerimientos; MDA; Usabilidad.

\begin{abstract}
The Internet has increased the development and use of applications in the web environment. However, not all web applications are successful in the market. Many systems are developed with usability flaws, which are very expensive to fix once the system is built. The usability of an information system determines the degree of satisfaction that a user experiences when interacting with it. For this reason, Usability is strongly linked to the software life cycle and it is necessary to incorporate it as a quality characteristic to take into account throughout the development process. One way to achieve this goal is through usability patterns. Information Systems Engineering has increased the use of patterns in the conceptual modeling of systems to ensure that no mistakes are made and use good learned practices. The following proposal shows a set of usability patterns that can be incorporated early into the conceptual modeling of systems, to guarantee the presence of certain usability criteria from the requirements elicitation stage.
\end{abstract}




\section{Introducción}

Un aspecto crítico en el desarrollo del software es la definición correcta de lo que se desea construir como sistema de información. La captura de requisitos adquiere un papel relevante, sobre todo cuando se plantean sistemas de alta complejidad donde la especificación de los requisitos es fundamental. De este modo, se observa la importancia de la elicitación de los requerimientos, puesto que permiten obtener un mejor conocimiento del dominio bajo estudio y contribuyen a mejorar el diseño y construcción de un producto, que responda a las necesidades de los usuarios (Qasim,I., Azam, F., Anwar, M. W., Tufail H., Qasim, T., 2018). La reestructuración de un sistema en una etapa tardía puede ser muy costosa y conducir al fracaso del proyecto por llegar tarde al mercado. Para mejorar esta situación, sería beneficioso que el conocimiento relacionado con la usabilidad se capture de una forma que pueda utilizarse para informar el diseño arquitectónico, de modo que las características de usabilidad sean tomadas en cuenta al principio del proceso de diseño. Una herramienta que la comunidad científica desarrolló para mejorar los procesos es la aplicación de patrones. En este contexto se analizará el desarrollo de patrones para la mejora de la usabilidad del software.

Este artículo tiene como objetivo hacer un acercamiento al estado en el que se encuentra la investigación sobre patrones de usabilidad, e identificar cuales patrones de aplicación temprana son de alto impacto en el modelado conceptual de los sistemas. Se trabaja con patrones puesto que contribuyen a no cometer errores por parte del diseñador del software y permite considerar aspectos que pueden haber sido pasado por alto.

El documento se organiza en secciones del siguiente modo: en la sección de Desarrollo se detallan distintos fundamentos teóricos relacionados con un patrón de usabilidad; luego se mencionan los principales patrones de Usabilidad cuyo origen es a partir de distintos trabajos científicos, para finalmente mostrar aquellos que tienen un gran impacto en el diseño del software y son aplicables a distintos escenarios partiendo desde la etapa de requisitos de software, mostrando un ejemplo de una aplicación particular. Finalmente se muestran los resultados obtenidos mediante un ejemplo y se brinda las conclusiones del análisis de la propuesta de trabajo.

\section{Desarrollo}

El concepto de patrón fue introducido por Alexander en 1977, en el dominio de la arquitectura. Se buscaba una solución basado en buenas prácticas a un problema considerado recurrente y específico. Cuando el concepto de patrón está referido al dominio de desarrollo del software, su concepción adopta diferentes propósitos. Implementar patrones en el proceso de desarrollo de un sistema es muy útil, porque contribuyen a diseñar mejor los modelos sin errores, estableciendo enlaces más consistentes entre la especificación de requerimientos y el modelado conceptual. El desarrollo de aplicaciones en entornos web ha suscitado la necesidad de incorporar aspectos de calidad relacionados con la usabilidad desde el inicio de la construcción de los sistemas de información. Este objetivo se puede lograr incorporando patrones (de usabilidad) en etapas tempranas del desarrollo de software, mediante la especificación de características inherentes a la Usabilidad en el modelado conceptual de los sistemas (Kaartinen, J., Palviainen J. \& Koskimies, K., 2007). La norma ISO/IEC 9126-1], considera a la usabilidad como un parámetro de calidad del software y la define como "la capacidad en que un producto de software puede ser entendido, aprendido y usado por determinados usuarios bajo ciertas condiciones en un contexto de uso específico". La norma que se adopta para analizar este aspecto de calidad (Usabilidad) es la ISO/IEC 25010.

\section{Patrones de Usabilidad}

Un patrón de usabilidad se define como una descripción de soluciones, que mejoran los atributos de Usabilidad en un determinado contexto y bajo ciertas condiciones. A partir de este concepto, se analizaron distintos tipos de patrones de Usabilidad, empleados en diversos contextos, momentos del desarrollo y de aplicación de los mismos. Los patrones de Usabilidad que interesaron analizar fueron aquellos que trataban a ciertos criterios usabilidad en etapas tempranas de desarrollo, o aquellos que eran tratados en metodologías que aplican el enfoque de desarrollo conducido por modelos (MDD) con patrones de Usabilidad (Herrera, J.L., Valdenebro, M.D., Juspian, M.A., Arroyo, A.T., \& Ordóñez, C.A., 2010). De los trabajos científicos que se analizaron, se obtuvo un listado de patrones que pueden ser especificados y reutilizados en la etapa de elicitación de 
requerimientos o en el proceso de diseño del modelado conceptual de un sistema. Dichos patrones brindan soporte y solucionan a distintos problemas específicos relacionados con criterios o subcriterios de usabilidad. Cabe destacar que dichos patrones no buscan solucionar temas relacionados de la interfaz del usuario, sino más bien con el comportamiento del sistema y específicamente de Usabilidad. Esto último permite capturar los mismos como requerimientos desde el punto de vista de la Ingeniería de Sistemas y validar con el usuario los criterios analizados (Ahmed Seffah, Taleb Mohamed, Halima Habieb-Mammar Alain Abran, 2008). A continuación, se detallan cada uno de los patrones de usabilidad evaluados, que se observan en la Tabla 1.

\begin{tabular}{|l|l|}
\hline \multicolumn{1}{|c|}{ Nombre } & \multicolumn{1}{c|}{$\begin{array}{c}\text { Propiedad de } \\
\text { Usabilidad que resuelve }\end{array}$} \\
\hline Cancel & Error management \\
\hline Context sensitive help & Guidance \\
\hline Different Languages & Accessibility \\
\hline History & Error management \\
\hline \multirow{2}{*}{ System feed back } & Explicit user control \\
\cline { 2 - 2 } & Provide feedback \\
\cline { 2 - 2 } & Long action feedback \\
\hline \multirow{2}{*}{ Undo } & Explicit user control \\
\cline { 2 - 2 } & Error management \\
\hline User profile & Adaptability \\
\hline User Input Error Prevention & Structured text entry \\
\hline \multirow{2}{*}{ Wizard } & Step by step solution \\
\hline Working data visualization & Visual consistency \\
\hline Reduce risk of errors & Visual consistency \\
\hline & Operability \\
\hline Dynamic presentation in user interface & Understandabüity \\
\cline { 2 - 2 } & Operability \\
\hline Progress indicator & User satisfaction \\
\hline \multirow{2}{*}{ Keep the user focused } & Visual consistency \\
\cline { 2 - 2 } & Understandabüity \\
\cline { 2 - 2 } & Operability \\
\hline \multirow{2}{*}{ Tabla de Patrones } & \\
\hline
\end{tabular}

Tabla 1: Tabla de Patrones de Usabilidad Analizados (ejemplo de un conjunto) .

Para poder evaluar la presencia de Usabilidad en un producto de software, la misma se descompone en subatributos que son medibles (Holger Röder, 2012). Esto permite vincular los subatributos con aspectos del diseño, que permiten verificar la presencia de la Usabilidad en los modelos conceptuales. El objetivo era vincular los atributos y los subatributos en los que se descompone la Usabilidad con su aplicación en el modelado conceptual mediante el empleo de patrones. Para ello, entonces, se buscó la relación que existe entre los patrones y los subcriterios de Usabilidad que pueden ser empleados en el modelado conceptual de aplicaciones web.

\section{Patrones de Usabilidad de Temprana Relevantes}

Es necesario realizar un vínculo entre las propiedades de usabilidad y los criterios. Para ello, se tomó como referencia la norma ISO/IEC 25010, que tiene en cuenta las propiedades de usabilidad desde dos puntos de vista: desde el punto de vista del producto y desde el punto de vista del proceso. Las propiedades de usabilidad se refieren a las características que debe satisfacer un producto de software para ser usable. Una vez identificado dichos atributos, se realizó un vínculo de cada propiedad con distintos patrones, que permitirían resolver problemas relacionados con propiedades específicas de la Usabilidad. Los patrones de Usabilidad proponen soluciones abstractas, que pueden ser incorporadas al modelado conceptual en forma temprana. Los patrones de Usabilidad propuestos representan a conceptos abstractos pero que son aplicables en el diseño. Para poder comprender dicha relación se estructura una tabla, donde se establece el vínculo entre los patrones, las propiedades o atributos que consideran a la usabilidad en forma temprana. 
Cabe destacar, sin embargo, que existen patrones que se emplean en etapas tempranas del desarrollo, durante la elicitación de requisitos y que son empleados en forma recurrente en los proyectos. Estos patrones se emplean para capturar un criterio de usabilidad específico. Se expone a continuación cuales son y una breve descripción de los mismos (ver Tabla 2). Dichos patrones, son considerados de alto impacto.

\begin{tabular}{|c|c|c|c|c|}
\hline $\begin{array}{l}\text { Característica de } \\
\text { Usabilidad }\end{array}$ & $\begin{array}{c}\text { Mecanismo } \\
\text { de } \\
\text { Usabilidad }\end{array}$ & Objetivo & $\begin{array}{l}\text { Alto impacto en la } \\
\text { funcionalidad del } \\
\text { software }\end{array}$ & $\begin{array}{l}\text { Autores que brindan } \\
\text { información sobre } \\
\text { esta función }\end{array}$ \\
\hline \multirow{4}{*}{$\begin{array}{c}\text { Feedback } \\
\text { (Retroalimentación) }\end{array}$} & $\begin{array}{l}\text { System } \\
\text { Status }\end{array}$ & $\begin{array}{l}\text { Para informar a los usuarios sobre el estado interno del } \\
\text { sistema. }\end{array}$ & \multirow{4}{*}{$\begin{array}{l}\text { Bass, Juristo. } \\
\text { (Bass L., John B., Kates. } \\
\text { J. , 2001; Juristo N., } \\
\text { Moreno A., Sánchez. } \\
\text { M., 2008) }\end{array}$} & \multirow{4}{*}{$\begin{array}{l}\text { Tidwell, Welie, Laasko, } \\
\text { Brighton, Coram }\end{array}$} \\
\hline & Interaction & $\begin{array}{l}\text { Para informar a los usuarios que el sistema ha registrado } \\
\text { una interacción del usuario, es decir, que el sistema ha } \\
\text { escuchado a los usuarios. }\end{array}$ & & \\
\hline & Warning & $\begin{array}{l}\text { Informar a los usuarios de cualquier acción con } \\
\text { consecuencias importantes. }\end{array}$ & & \\
\hline & $\begin{array}{l}\text { Long Action } \\
\text { Feedback } \\
\end{array}$ & $\begin{array}{l}\text { Para informar a los usuarios que el sistema está } \\
\text { procesando una acción que llevará un tiempo completar }\end{array}$ & & \\
\hline \multirow{4}{*}{$\begin{array}{c}\text { Undo/Cancel } \\
\text { (Deshacer/Cancelar) }\end{array}$} & Global Undo & Para deshacer acciones del sistema en varios niveles & \multirow{4}{*}{$\begin{array}{l}\text { Bass, Juristo. } \\
\text { (Bass L., John B., Kates } \\
\text { J. , 2001; Juristo N., } \\
\text { Moreno A., Sánchez. } \\
\text { M., 2008) }\end{array}$} & \multirow{4}{*}{$\begin{array}{l}\text { Brighton, Tidwell, } \\
\text { Welie, Laasko }\end{array}$} \\
\hline & \begin{tabular}{|c|} 
Object- \\
Specific \\
Undo \\
\end{tabular} & Para deshacer varias acciones en un objeto & & \\
\hline & $\begin{array}{c}\text { Abort } \\
\text { Operation } \\
\end{array}$ & Para cancelar la ejecución de un comando o una aplicación & & \\
\hline & $\begin{array}{c}\text { Go Back to a } \\
\text { Safe State }\end{array}$ & $\begin{array}{l}\text { Para volver a un estado particular en una secuencia de } \\
\text { ejecución de comando }\end{array}$ & & \\
\hline $\begin{array}{c}\text { Form/Field } \\
\text { Validation } \\
\text { (Validación de } \\
\text { Formulario/Campo) }\end{array}$ & $\begin{array}{l}\text { Structured } \\
\text { Text Entry }\end{array}$ & $\begin{array}{l}\text { Para ayudar a evitar que el usuario cometa errores de } \\
\text { entrada de datos. }\end{array}$ & $\begin{array}{l}\text { Bass, Juristo. } \\
\text { (Bass L., John B., Kates. } \\
\text { J. , 2001; Juristo N., } \\
\text { Moreno A., Sánchez. } \\
\text { M., 2008) }\end{array}$ & Brighton, Tidwell \\
\hline $\begin{array}{l}\text { Wizard } \\
\text { (Asistente) }\end{array}$ & $\begin{array}{l}\text { Step-by-Step } \\
\text { Execution }\end{array}$ & $\begin{array}{l}\text { Para ayudar a realizar tareas que requieren diferentes } \\
\text { pasos con la entrada del usuario }\end{array}$ & $\begin{array}{l}\text { Bass, Juristo. } \\
\text { (Bass L., John B., Kates. } \\
\text { J. , 2001; Juristo N., } \\
\text { Moreno A., Sánchez. } \\
\text { M., 2008) }\end{array}$ & Welie \\
\hline \multirow{3}{*}{$\begin{array}{c}\text { User Profile } \\
\text { (Perfil de Usuario) }\end{array}$} & Preferences & $\begin{array}{l}\text { Para registrar las opciones de cada usuario para trabajar } \\
\text { con el sistema a nivel funcional }\end{array}$ & \multirow{3}{*}{$\begin{array}{l}\text { Bass, Juristo. } \\
\text { (Bass L., John B., Kates. } \\
\text { J. , 2001; Juristo N., } \\
\text { Moreno A., Sánchez. } \\
\text { M., 2008) }\end{array}$} & \multirow{3}{*}{ Welie, Tidwell } \\
\hline & \begin{tabular}{|c|} 
Personal \\
Object Space \\
\end{tabular} & $\begin{array}{l}\text { Para registrar las opciones de cada usuario para trabajar } \\
\text { con el sistema a nivel de interfaz. }\end{array}$ & & \\
\hline & Favourites & Para registrar ciertos lugares de interés para el usuario. & & \\
\hline $\begin{array}{l}\text { Help } \\
\text { (Ayuda) }\end{array}$ & $\begin{array}{l}\text { Multilevel } \\
\text { Help }\end{array}$ & $\begin{array}{l}\text { Proporcionar diferentes niveles de ayuda para diferentes } \\
\text { usuarios. }\end{array}$ & \begin{tabular}{l}
\multicolumn{1}{c}{ Bass, Juristo. } \\
(Bass L., John B., Kates. \\
J. , 2001; Juristo N., \\
Moreno A., Sánchez. \\
M., 2008)
\end{tabular} & Tidwell \\
\hline
\end{tabular}

Tabla 2: Patrones de Elicitación de Usabilidad

Un patrón de usabilidad se define como una descripción de soluciones, que mejoran los atributos de usabilidad. Las mejoras que ofrecen los patrones presentados a los aspectos de usabilidad, se pueden aplicar posteriormente a las interfaces de los usuarios. Esto da origen a que los mismos se puedan asociar posteriormente con patrones de interfaz o patrones de diseño de interacción. (Garzotto F., Paolini P., Bolchini D., Valenti S., 1999; Folmer, 2003) 


\section{Descripción de un Patrón de Usabilidad}

A continuación, se describirá como se describirán cada patrón arquitectónico, desde el punto de vista de los escenarios, describiendo la especificación de cada una de las partes que contendrá el mismo. Esto ayudará a describir cada patrón de usabilidad y posibilita la construcción de un catálogo de patrones que resultan útil al diseñador. A su vez, su descripción posibilita la selección en forma inequívoca del patrón, para el problema que se intenta solucionar. La inclusión de patrones de usabilidad en etapas tempranas del diseño del software no es habitual (Ver Gráfico 1). Sin embargo, en los últimos años el desarrollo de aplicaciones web que emplean patrones ha sido incremental, puesto que garantizan no cometer errores e incluyen aspectos de buenas prácticas que pueden no haber relevados en la etapa de elicitación de requerimientos. Se muestra a continuación su estructura:

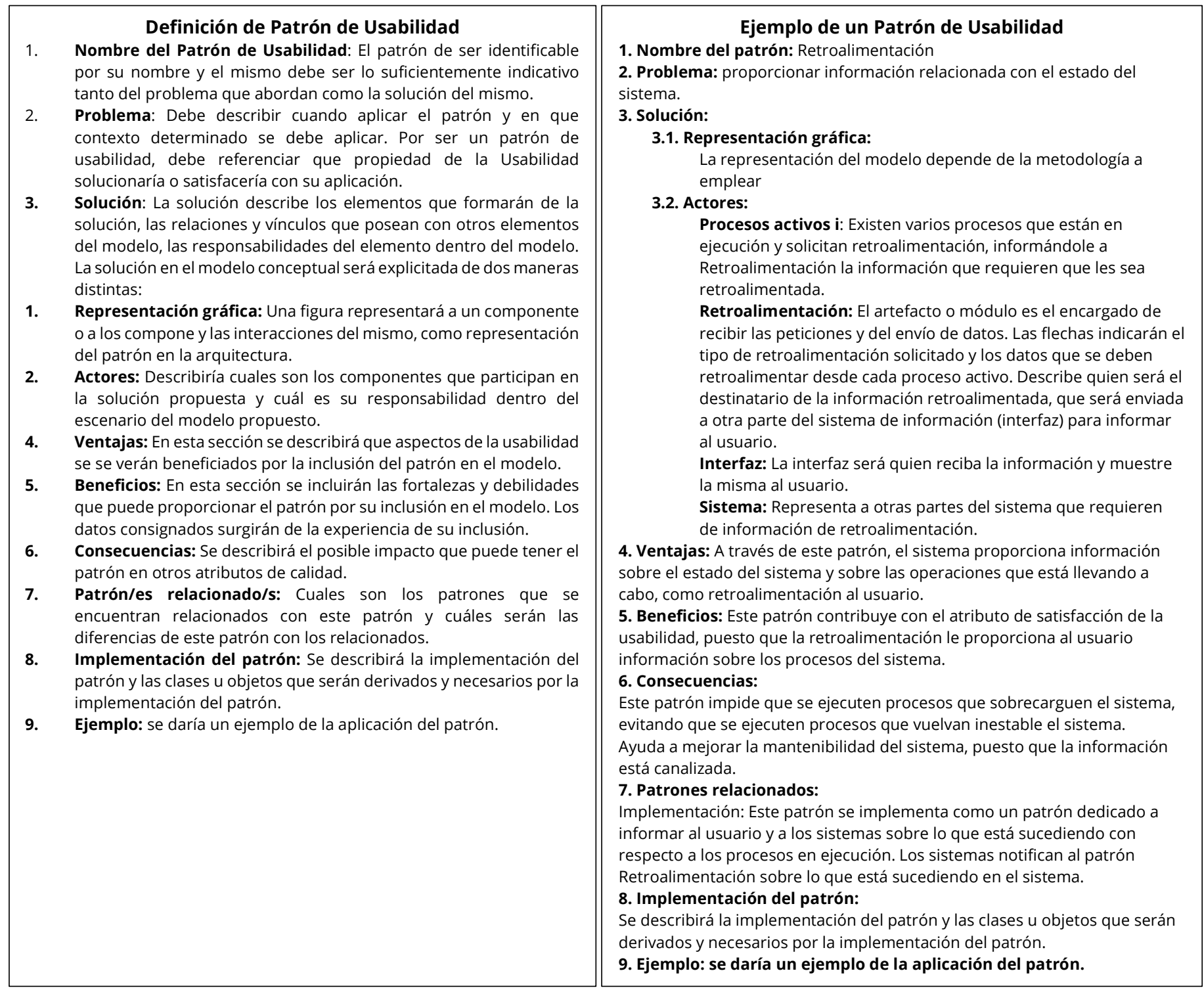

Gráfico 1: Ejemplo de la Definición de un Patrón de Usabilidad Temprana.

\section{Resultados}

Una vez comprendidos los patrones que pueden emplearse en el modelado conceptual de los sistemas, se elaboró un catálogo de patrones, que brindan al analista de sistemas de información dos aspectos como ventajas: a) la facilidad de poder seleccionar un patrón de usabilidad, que está en función de un atributo del mismo que se desea mejorar en el modelo conceptual para un uso específico; b) asimismo, además, brinda información sobre 
el impacto que puede provocar sobre otros atributos la inclusión de dicho patrón en el modelo. Estos aspectos representan una contribución importante para quien diseña sistemas.

\section{Conclusión}

Los patrones de usabilidad son empleados en el modelado conceptual como una solución para mejorar ciertos aspectos de calidad, que se carecen o necesitan ser mejorados en el modelado conceptual de los sistemas. La generación de patrones de usabilidad conlleva a plantear la necesidad de establecer una guía de buenas prácticas para la selección de patrones desde un catálogo, el cual debe reunir ciertas características tales como: a) poder seleccionar un patrón de usabilidad de forma precisa, ya sea por los atributos que el patrón intenta solucionar y qué impacto puede producir su introducción en el modelo; b) como emplear el patrón de usabilidad seleccionado, en un modelo conceptual de un dominio determinado.

La incorporación de forma temprana, implica que los requerimientos de Usabilidad deben ser capturados desde la etapa de elicitación de requerimientos. Esto trae aparejado la inclusión de otras propiedades de usabilidad debido a que muchas de estas propiedades están vinculadas unas con otras. La idea de considerar la usabilidad desde la etapa de elicitación de requerimientos influirá en el desarrollo y diseño de la arquitectura del software, y en su posterior implementación tras la adopción de un lenguaje de programación. Esto contribuirá a disminuir los tiempos de reprocesos, disminuirá los costos y ayudará a obtener un software de calidad.

\section{Referencias}

Ahmed Seffah, Taleb Mohamed, Halima Habieb-Mammar, Alain Abran, "Reconciling usability and interactive system architecture using patterns”, Journal of Systems and Software Volume 81, Issue 11, Pages 1845-1852, 2008

Bass L., John B., Kates. J. Achieving Usability Through Software Architecture. Technical Report. CMU/SEI-2001-TR005, March 2001.

Brighton, P. Usability Pattern Collection. Http://www.cmis.brighton.ac.uk.research/patterns

Folmer, E. (2003). Usability Patterns in Software Architecture.

Garzotto F., Paolini P., Bolchini D., Valenti S. (1999) “Modeling-by-Patterns” of Web Applications. In: Chen P.P., Embley D.W., Kouloumdjian J., Liddle S.W., Roddick J.F. (eds) Advances in Conceptual Modeling. ER 1999. Lecture Notes in Computer Science, vol 1727. Springer, Berlin, Heidelberg

Holger Röder, "Specifying usability features with patterns and templates", First International Workshop on Usability and Accessibility Focused Requirements Engineering (UsARE), 2012

Herrera, J.L., Valdenebro, M.D., Juspian, M.A., Arroyo, A.T., \& Ordóñez, C.A. (2010). Architectural patterns regarding web application domain usability

Juristo N., Moreno A., Sánchez. M. Techniques and Patterns for Architecture-Level Usability Improvements. Deliverable 3.4. STATUS. project. Http://www.ls.fi.upm.es/status May 2003.

Kaartinen J., Palviainen, J, Koskimies, K., "A Pattern-Driven Process Model for Quality-Centered Software Architecture Design - A Case Study on Usability-Centered Design”, Australian Software Engineering Conference (ASWEC'07), 2007

Qasim,I., Azam, F., Anwar, M. W., Tufail H., Qasim, T. "Mobile User Interface Development Techniques: A Systematic Literature Review," 2018 IEEE 9th Annual Information Technology, Electronics and Mobile Communication Conference (IEMCON), Vancouver, BC, 2018, pp. 1029-1034, doi: 10.1109/IEMCON.2018.8614764.

Laasko, S. A.. User Interface Designing Patterns, 2003. http://www.cs.helsinki.fi/u/salaakso/patterns/index tree.html

Tidwell, J. The Case for HCI Design Patterns. Http://www.mit.edu/jdidwell/common ground onefile.htm

Tidwell, J. UI Patterns and Techniques. Http://time-tripper.com/uipatterns

Welie, M. Van. The Amsterdam Collection of Patterns in User Interface Design. Http://www.welie.com . 